OU-HET 202

LMU-TPW 94-13

October, 1994

\title{
On Bogoliubov Transformation of Scalar Wave Functions in De Sitter Space
}

\author{
Haru-Tada Sato 0 \\ Sektion Physik der Universität München, \\ Theresienstrasse 37, D-80333 München, Germany \\ Hisao Suzuki i \\ Department of Physics, Osaka University \\ Toyonaka, Osaka 560, Japan
}

\begin{abstract}
We discuss the Bogoliubov transformation of the scalar wave functions caused by the change of coordinates in 4 dimensional de Sitter space. It is shown that the exact Bogoliubov coefficients can be obtained from the global coordinates to the static coordinates where there exist manifest horizon. We consider two type of global coordinates. In one global coordinates, it is shown that the Bogoliubov transformation to the static coordinates can be expressed by the discontinuous integral of Weber and Schafheitlin. The positive and negative energy states in the global coordinates degenerate in the static coordinates. In the other global coordinates, we obtain the Bogoliubov coefficients by using the analytic continuation of the hypergeometric functions in two variables.

\footnotetext{
${ }^{*}$ Fellow of the Japan Society for the Promotion of Science, On leave of absence from Osaka University e-mail address: hsato@phys.wani.osaka-u.ac.jp

$\dagger$ †e-mail address: suzuki@phys.wani.osaka-u.ac.jp
} 
We also discuss the relation between two type of global coordinates and find an integral relation between the mode functions. 


\section{Introduction}

Quantum field theories in curved space time have been investigated extensively. One of the celebrating result is the existence of Hawking radiations [1]. Although true nature of the radiation should be revealed when we include the effect of the back reaction, some of the difficulty of the treatment arise from the fact that the quantum effect such as in Schwarzschild metric cannot be expressed by well-known special functions where analytic properties can be extracted. A simple example of the metric which possess horizons arises in the cosmological situation such as de Sitter space. In this metric, it was shown that the wave functions of the scalar fields can be solved by special functions in several coordinates 2] [3] [5]. Hawking radiation has been analyzed by several arguments. One way is the use of Kruskal coordinates and the analytic continuation of the Green functions. [6]. Later, an explicit scattering state has been considered using Unruh's observation [7] and Gibbons-Hawking vacuum. [4] The other interesting method is to consider the Bogoliubov transformation of the mode functions between the static coordinates and the global coordinates where there is no loss of information. [8] Namely, we can define the vacuum in the static coordinates by use of the vacuum of the global coordinates. this program has been discussed by using high frequency approximation and find the factor showing the existence of the radiation. In principle, the de Sitter space seems to be one of the rare example that we can obtain the exact Bogoliubov coefficients analytically because the modes functions in both coordinates can be expressed by special functions. The coefficients should contain the information of the effects of various parameter such as masses to Hawking radiation without using S-wave approximation.

Our aim of this paper is to evaluate the Bogoliubov coefficients of the transformation exactly. We treat two type of globally defined coordinates where there is no horizon. We obtain the transformation functions of the scalar wave functions to the static coordinates. The Bogoliubov transformation from one of the global coordinates can be expressed by the so-called discontinuous integral of Weber and Schafheitlin type 9. Using this formula we show that the we cannot define the well defined vacua in the static coordinates. 
Actually, the non-analyticity of mode functions is known for lower dimensional examples such as Rindler space. Our case must be a four-dimensional example of such known phenomena. As for the Bogoliubov coefficient from the other global coordinates, we have to use the analytic continuation of the mode functions inside the horizon. We evaluate the coefficients in terms of the hypergeometric functions in two variables. For this purpose, we prove some formula for these functions.

We also discuss the relation between the mode functions between two type of the global coordinates and find an integral formula. We comment on the relation of our results and the previous calculation. It seems interesting that these transformations can be expressed by various integral formula including bi-linear of the special functions.

\section{Notation}

The coordinates of de Sitter space are obtained by various parameterization of the flat space-time of the coordinates $z_{a} a=0,2, \ldots, 4$ satisfying

$$
-z_{0}^{2}+z_{1}^{2}+z_{2}^{2}+z_{3}^{2}+z_{4}^{2}=1
$$

where we have normalized the radius to one. A global parameterization of this coordinates is given by

$$
\begin{aligned}
& z_{0}=\sinh t_{1}+\frac{1}{2} e^{t_{1}} r_{1}^{2}, \\
& z_{4}=\cosh t_{1}-\frac{1}{2} e^{t_{1}} r_{1}^{2} \\
& z_{1}=e^{t_{1}} r_{1} \sin \theta \sin \phi \\
& z_{2}=e^{t_{1}} r_{1} \sin \theta \cos \phi \\
& z_{3}=e^{t_{1}} r_{1} \cos \theta
\end{aligned}
$$

The conformal time is given by

$$
\eta_{1}=-e^{-t_{1}}, \quad-\infty<\eta_{1}<0
$$


by which the metric is expressed as

$$
(d s)^{2}=\frac{1}{\eta_{1}^{2}}\left[d \eta_{1}^{2}-d r_{1}^{2}-r_{1}^{2}\left(d \theta^{2}+\sin \theta^{2} d \phi^{2}\right)\right] .
$$

We shall call this coordinates as type-I global coordinates. In this coordinates, the KleinGordon equation for a scalar field $g^{\mu \nu} \nabla_{\mu} \nabla_{\nu} \phi+m^{2} \phi=0$ can be solved in terms of the Bessel functions as [2]

$$
\phi_{k, l, m}^{I, \pm \nu}\left(\eta_{1}, r_{1}, \theta, \phi\right)=\left(-k \eta_{1}\right)^{3 / 2} J_{ \pm \nu}\left(-k \eta_{1}\right)\left(k r_{1}\right)^{-1 / 2} J_{l+1 / 2}\left(k r_{1}\right) Y_{l, m}(\theta, \phi)
$$

where we have chosen $J_{ \pm \nu}$ to be two independent solutions for the time $\eta_{1} . Y_{l, m}(\theta, \phi)$ is the spherical harmonics and $\nu=\left(9 / 4-m^{2}\right)^{1 / 2}$.

Another global coordinates is given by

$$
\begin{aligned}
z_{0} & =\sinh t_{2}, \\
z_{4} & =\cosh t_{2} \cos \chi, \\
z_{1} & =\cosh t_{2} \sin \chi \sin \theta \sin \phi, \\
z_{2} & =\cosh t_{2} \sin \chi \sin \theta \cos \phi, \\
z_{3} & =\cosh t_{2} \sin \chi \cos \theta,
\end{aligned}
$$

which we denote by type-II global coordinates. We introduce the conformal time in this system as

$$
\tan \frac{\eta_{2}}{2}=e^{t_{2}}, \quad 0 \leq \eta_{2}<\pi
$$

so that the metric is expressed as

$$
d s^{2}=\sin ^{-2} \eta_{2}\left[d \eta_{2}^{2}-d \chi^{2}-\sin ^{2} \chi\left(d \theta^{2}+\sin ^{2} \theta d \phi^{2}\right)\right]
$$

In this coordinates, the mode function are expressed as 3 [ [0.

$$
\phi_{N, l, m}^{I I, \pm \nu}\left(\eta_{2}, \chi, \theta, \phi\right)=\left(\sin \eta_{2}\right)^{3 / 2} P_{N+1 / 2}^{ \pm \nu}\left(\cos \eta_{2}\right) C_{N-l}^{l+1}(\cos \chi)(\sin \chi)^{l} Y_{l, m}(\theta, \phi)
$$

where $P_{\nu}^{\mu}(z)$ is the associated Legendre function and $C_{n}^{\nu}(z)$ denotes Gegenbauer's polynomial. We can also choose $P_{N+\frac{1}{2}}^{\nu}\left(\cos \eta_{2}\right)$ and $Q_{N+\frac{1}{2}}^{\nu}\left(\cos \eta_{2}\right)$ as two independent solutions for the mode functions with respect to the variable $\eta_{2}$. 
As the third coordinates, we consider the static coordinates defined by

$$
\begin{aligned}
& z_{0}=\left(1-r^{2}\right)^{1 / 2} \sinh t, \\
& z_{4}=\left(1-r^{2}\right)^{1 / 2} \cosh t, \\
& z_{1}=r \sin \theta \sin \phi, \\
& z_{2}=r \sin \theta \cos \phi, \\
& z_{3}=r \cos \theta .
\end{aligned}
$$

This coordinates covers the half of the de Sitter space with $z_{0}+z_{4}>0$. In this coordinates, the metric can be written as

$$
d s^{2}=\left(1-r^{2}\right) d t^{2}-\left(1-r^{2}\right)^{-1} d r^{2}-r^{2}\left(d \theta^{2}+\sin \theta^{2} d \phi^{2}\right) .
$$

The line element possesses a coordinates singularity at $r=1$, which is the event horizon for an observer at $r=0$. The solution of the scalar wave functions are written by the hypergeometric functions $\mathbb{4}$;

$$
\phi_{w, l, m}^{S,( \pm)}(t, r, \theta, \phi)=e^{ \pm i \omega t} r^{l}\left(1-r^{2}\right)^{i \omega / 2} F\left(a, b ; c ; r^{2}\right),
$$

where

$$
\begin{aligned}
a, b & =\frac{1}{2} c+\frac{i \omega}{2} \pm \frac{\nu}{2}, \\
c & =l+\frac{3}{2}
\end{aligned}
$$

and $F(a, b ; c ; z)$ is the hypergeometric function. We have omitted the radial functions which diverges at $r=0$ as in (2.9).

\section{Bogoliubov transformation from the type-I coor- dinates to the static coordinates}

We are going to argue the relations among these coordinates. To begin with, we consider the transformation between the static coordinates and the type-I global coordinates. By 
comparing (2.2) and (2.10), we can find the relation of the coordinates $\eta_{1}, r_{1}$ and $t, r$ as

$$
\begin{aligned}
r & =-\frac{r_{1}}{\eta_{1}} \\
t & =\frac{1}{2} \ln \left(\eta_{1}^{2}-r_{1}^{2}\right)
\end{aligned}
$$

We take the Bogoliubov transformation from (2.5) to (2.12) to be of the form;

$$
\phi_{\omega, l, m}^{S( \pm)}(t, r)=\int_{0}^{\infty} a_{\omega}^{( \pm)}(k, \nu) \phi_{k, l, m}^{I, \nu}\left(\eta_{1}, r_{1}\right) d k
$$

where $a(k)$ is a function to be determined. The coefficient $a^{ \pm}$as a function of $k$ can be obtained by considering the Killing vector $\frac{\partial}{\partial t}$. Note that the functions $\phi^{S( \pm)}$ are the eigenfunction of this killing vector which can be written in the global coordinates as

$$
\frac{\partial}{\partial t}=\eta_{1} \frac{\partial}{\partial \eta_{1}}+r \frac{\partial}{\partial r}
$$

Since the mode function (2.5) is the function of $k \eta_{1}$ and $k r_{1}$, the action of the killing vector to (3.2) is given by $k \frac{\partial}{\partial k}$ in the integral. By using the partial integration, we get the following equation for $a(k)$;

$$
-\frac{d}{d k}\left(k a^{ \pm}(k)\right)= \pm i w a^{ \pm}(k)
$$

The solution of this equation is simply given by

$$
a^{( \pm)}(k)=c k^{\mp i w-1}
$$

where $c$ is a coefficient independent of $k$. The integral of the form (3.2) with (3.5) is known as the discontinuous integral of Weber and Schafheitlin 9], which is

$$
\begin{gathered}
2^{\rho} a^{-\mu} b^{\mu-\rho+1} \frac{\Gamma(\mu+1) \Gamma\left(\frac{1}{2}+\frac{1}{2} \nu+\frac{1}{2} \rho-\frac{1}{2} \mu\right)}{\Gamma\left(\frac{1}{2}+\frac{1}{2} \nu+\frac{1}{2} \mu-\frac{1}{2} \rho\right)} \int_{0}^{\infty} J_{\mu}(a t) J_{\nu}(b t) t^{-\rho} \\
F\left(\frac{1}{2}+\frac{1}{2} \nu+\frac{1}{2} \mu-\frac{1}{2} \rho, \frac{1}{2}+\frac{1}{2} \mu-\frac{1}{2} \nu-\frac{1}{2} \rho ; \nu+1 ; \frac{a^{2}}{b^{2}}\right) .
\end{gathered}
$$

By comparing this integral formula to (3.2), we have

$$
\phi^{S,-}=2^{-i \omega} \frac{\Gamma\left(l+\frac{3}{2}\right) \Gamma\left(\frac{1}{2}\left(1 \pm \nu-i \omega-l-\frac{1}{2}\right)\right)}{\Gamma\left(\frac{1}{2}\left( \pm \nu+l+\frac{1}{2}+i \omega\right)\right)} \int_{0}^{\infty} d k k^{i w-1} \phi^{I, \pm \nu}
$$


Note that the two independent solutions in global coordinates are transformed to an identical mode function in the static coordinates. Namely, the positive and negative energy wave functions are degenerate in the static coordinates. Correspondingly, another mode function $\phi^{+}$in the static coordinates can not be represented by the Bogoliubov transformation from the type-I global coordinates. This mode can be obtained by the analytic continuation from inside the horizon $r^{2}>1$.

\section{The type-II to the static coordinates}

We next consider the Bogoliubov transformation the type-II coordinates and the static coordinates, which is discussed in ref. [8] using approximations. We consider the Bogoliubov transformation of the form

$\phi^{S, \pm}(t, r, \theta, \varphi)=\sum_{N \geq l}\left[A_{N}^{ \pm} P_{N+\frac{1}{2}}^{\nu}\left(\cos \eta_{2}\right)+B_{N}^{ \pm} Q_{N+\frac{1}{2}}^{\nu}\left(\cos \eta_{2}\right)\right] C_{N-l}^{l+1}(\cos \chi)(\sin \chi)^{l} Y_{l, m}(\theta, \varphi)(4.1)$

The change of variables is given by

$$
\begin{aligned}
t & =\frac{1}{2}[\ln (x-y)-\ln (x+y)] \\
r & =\left(\frac{1-x^{2}}{1-y^{2}}\right)^{1 / 2}
\end{aligned}
$$

where $x=\cos \chi, y=\cos \eta_{2}$. As in the previous consideration, we can construct a recursion relation of the coefficients $A_{N}^{ \pm}$and $B_{N}^{ \pm}$by considering the killing vector

$$
\frac{\partial}{\partial t}=-\left(1-x^{2}\right) y \frac{\partial}{\partial x}-\left(1-y^{2}\right) x \frac{\partial}{\partial y}
$$

Expressing the Gegenbauer polynomial in terms of the Legendre functions and using recursion relations of the Legendre functions both for variables $x$ and $y$, we obtain the following difference equations;

$$
\begin{aligned}
\frac{\left(N+\nu+\frac{3}{2}\right)(N+l+2)}{2(N+2)} C_{N+1}^{ \pm} & =\mp i \omega C_{N}^{ \pm}+\frac{\left(N-\nu+\frac{1}{2}\right)(N-l)}{2 N} C_{N-1}^{ \pm}, \\
C_{l+1}^{ \pm} & =\mp i \omega \frac{l+2}{(l+1)\left(l+\nu+\frac{3}{2}\right)} C_{l}^{ \pm}
\end{aligned}
$$


where $C_{N}^{ \pm}$denotes both $A_{N}^{ \pm}$and $B_{N}^{ \pm}$. Hence, after obtaining one of the coefficients $A_{l}^{ \pm}$ and $B_{l}^{ \pm}$, we can determine the other coefficients through these recursion formulae (4.4) and (4.5). Note also that the ratio of $A_{N}$ and $B_{N}$ does not depend on $N$ because both coefficients obey an identical recursion relation. We can determine the coefficient for $N=l$ by using the orthogonality of Gegenbauer's polynomial and find the mode function $G_{l}^{ \pm}\left(\eta_{2}\right) \equiv A_{l}^{ \pm} P_{l+\frac{1}{2}}^{\nu}\left(\cos \eta_{2}\right)+B_{l}^{ \pm} Q_{l+\frac{1}{2}}^{\nu}\left(\cos \eta_{2}\right)$ can be expressed by

$$
\begin{aligned}
G_{l}^{ \pm}\left(\eta_{2}\right)= & \frac{\Gamma(l+2)}{\pi^{\frac{1}{2}} \Gamma\left(l+\frac{3}{2}\right)} \int_{0}^{\pi} \phi^{S, \pm}(t, r)(\sin \chi)^{l+2} \\
= & \frac{\Gamma(l+2)}{\pi^{\frac{1}{2}} \Gamma\left(l+\frac{3}{2}\right)}\left(\sin \eta_{2}\right)^{-l-i w} \int_{0}^{\pi} d \chi\left(\cos \eta_{2} \mp \cos \chi\right)^{i \omega}(\sin \chi)^{2 l+2} F\left(a, b ; c ; \frac{\sin ^{2} \chi}{\sin ^{2} \eta_{2}}\right) \\
= & \frac{\Gamma(l+2)}{\pi^{\frac{1}{2}} \Gamma\left(l+\frac{3}{2}\right)}\left(\sin \eta_{2}\right)^{-l-i w} \int_{0}^{\frac{\pi}{2}} d \chi\left[\left(\cos \eta_{2}+\cos \chi\right)^{i \omega}+\left(\cos \eta_{2}-\cos \chi\right)^{i \omega}\right] \\
& \times(\sin \chi)^{2 l+2} F\left(a, b ; c ; \frac{\sin ^{2} \chi}{\sin ^{2} \eta_{2}}\right),
\end{aligned}
$$

In order to find explicit Bogoliubov coefficients, we have to use analytic continuation because the integral over $\chi$ does not preserve the condition $r^{2}<1$. The integral formula of this type can not be found in the literature so we prove some formula. For this purpose, making use of the following representation 10;

$$
\left(1+z^{\frac{1}{2}}\right)^{-2 a}+\left(1-z^{\frac{1}{2}}\right)^{-2 a}=2 F\left(a, a+\frac{1}{2} ; \frac{1}{2} ; z\right)
$$

We can express the RHS of (4.6) in the following form;

$$
\begin{aligned}
G_{l}\left(\eta_{2}\right)= & \frac{2 \Gamma(l+2)}{\pi^{\frac{1}{2}} \Gamma\left(l+\frac{3}{2}\right)}\left(\sin \eta_{2}\right)^{-l-i w}\left(\cos \eta_{2}\right)^{i \omega} \\
& \times \int_{0}^{\frac{\pi}{2}} d \chi(\sin \chi)^{2 l+2} F\left(-\frac{i \omega}{2},-\frac{i \omega}{2}+\frac{1}{2} ; \frac{1}{2} ; \frac{\cos ^{2} \chi}{\cos ^{2} \eta_{2}}\right) F\left(a, b ; c ; \frac{\sin ^{2} \chi}{\sin ^{2} \eta_{2}}\right) \\
= & \frac{\Gamma(l+2)}{\pi^{\frac{1}{2}} \Gamma\left(l+\frac{3}{2}\right)}\left(1-x^{2}\right)^{\frac{1}{2}(-l-i w)} x^{i \omega} \\
& \times \int_{0}^{1} d t t^{-\frac{1}{2}}(1-t)^{c-1} F\left(-\frac{i \omega}{2},-\frac{i \omega}{2}+\frac{1}{2} ; \frac{1}{2} ; \frac{t}{x^{2}}\right) F\left(a, b ; c ; \frac{1-t}{1-x^{2}}\right)
\end{aligned}
$$

where we have set $\cos ^{2} \chi=t$ and $\cos \eta_{2}=x$. Note that the integral of the form can be expressed by the hypergeometric functions in two variables [11] as

$$
G_{l}=x^{i w}\left(1-x^{2}\right)^{-\frac{1}{2}(i \omega+l)} F_{3}\left(-\frac{i \omega}{2}, a,-\frac{i \omega}{2}+\frac{1}{2}, b ; c+\frac{1}{2} ; \frac{1}{x^{2}}, \frac{1}{1-x^{2}}\right),
$$


where the function $F_{3}\left(\alpha, \alpha^{\prime}, \beta, \beta^{\prime}, \gamma, x, y\right)$ is defined by 13

$$
F_{3}\left(\alpha, \alpha^{\prime}, \beta, \beta^{\prime}, \gamma, x, y\right)=\sum \frac{(\alpha)_{m},\left(\alpha^{\prime}\right)_{n}(\beta)_{m}\left(\beta^{\prime}\right)_{n}}{(\gamma)_{m+n} m ! n !} x^{m} y^{n}
$$

and $(\alpha)_{n}$ denotes $\Gamma(\alpha+n) / \Gamma(\alpha)$. We analytically continue this function to the whole $x$-plane. Since the true value of $x$ stays on the cut, we consider the value to be

$$
G_{l}(x)=\frac{1}{2}\left(G_{l}(x+i 0)+G_{l}(x-i 0)\right)
$$

which guarantee the validity of this integral around $x=0$.

We are now going to obtain the Bogoliubov coefficient. We first perform some analytic continuations to rewrite the $F_{3}$ in (4.9) into ${ }_{2} F_{1}$. To begin with, we analytically continue the function $F_{3}\left(\alpha, \alpha^{\prime}, \beta, \beta^{\prime}, \gamma, x, y\right)$ into the function $F_{2}$ as 12

$$
\begin{aligned}
& F_{3}\left(\alpha, \alpha^{\prime}, \beta, \beta^{\prime}, \gamma, \frac{1}{x^{2}}, \frac{1}{1-x^{2}}\right) \\
= & \sum \frac{\Gamma(\gamma) \Gamma(\rho-\lambda) \Gamma(\sigma-\mu)}{\Gamma(\rho) \Gamma(\sigma) \Gamma(\gamma-\Lambda-\mu)}\left(-x^{-2}\right)^{-\lambda}\left(x^{2}-1\right)^{-\mu} \\
& \times F_{2}\left(\lambda+\mu+1-\gamma, \lambda, \mu, \lambda-\rho+1, \mu-\sigma+1 ; x^{2}, 1-x^{2}\right),
\end{aligned}
$$

where the sum consists of four terms in which $\lambda, \rho$ take $\alpha, \beta ; \beta, \alpha$ and $\mu, \rho$ is $\alpha^{\prime}, \beta^{\prime} ; \beta^{\prime}, \alpha^{\prime}$, and $F_{2}\left(\alpha, \beta, \beta^{\prime}, \gamma, \gamma^{\prime}, x, y\right)$ is defined by 13

$$
F_{2}\left(\alpha, \beta, \beta^{\prime}, \gamma, \gamma^{\prime}, x, y\right)=\sum \frac{(\alpha)_{m+n}(\beta)_{m}\left(\beta^{\prime}\right)_{n}}{(\gamma)_{m}\left(\gamma^{\prime}\right)_{n} m ! n !} x^{m} y^{n}
$$

We then transform this function by using the formula[14];

$$
F_{2}\left(\alpha, \beta, \beta^{\prime}, \gamma, \gamma^{\prime} ; x, y\right)=(1-x)^{-\alpha} F_{2}\left(\alpha, \gamma-\beta, \beta^{\prime}, \gamma, \gamma^{\prime} ; \frac{x}{x-1}, \frac{y}{1-x}\right)
$$

which implies

$$
\begin{aligned}
& F_{3}\left(\alpha, \alpha^{\prime}, \beta, \beta^{\prime}, \gamma ; \frac{1}{x^{2}}, \frac{1}{1-x^{2}}\right) \\
= & \sum e^{-\pi i \lambda} \cos \pi \mu \frac{\Gamma(\gamma) \Gamma(\rho-\lambda) \Gamma(\sigma-\mu)}{\Gamma(\rho) \Gamma(\sigma) \Gamma(\gamma-\Lambda-\mu)} x^{2 \lambda}\left(1-x^{2}\right)^{\gamma-\lambda-1} \\
& \times F_{2}\left(\lambda+\mu+1, \lambda, \mu, \lambda-\rho+1 ; \frac{x^{2}}{x^{2}-1}, 1\right)
\end{aligned}
$$


By the definition of $F_{2}$, we find

$$
\begin{aligned}
& F_{2}\left(\alpha, \beta, \beta^{\prime}, \gamma, \gamma^{\prime} ; x, 1\right) \\
& \quad=\frac{\Gamma\left(\gamma^{\prime}\right) \Gamma\left(\gamma^{\prime}-\alpha-\beta^{\prime}\right)}{\Gamma\left(\gamma^{\prime}-\beta^{\prime}\right) \Gamma\left(\gamma^{\prime}-\alpha\right)}{ }_{3} F_{2}\left(\alpha, \beta, \alpha-\gamma^{\prime}+1 ; \gamma, \alpha+\beta^{\prime}-\gamma^{\prime}+1 ; x\right),
\end{aligned}
$$

where ${ }_{3} F_{2}(\alpha, \beta, \gamma ; \delta, \lambda ; x)$ is defined by

$$
{ }_{3} F_{2}(\alpha, \beta, \gamma ; \delta, \lambda ; x)=\sum_{m} \frac{(\alpha)_{m}(\beta)_{m}(\gamma)_{m}}{(\delta)_{m}(\lambda)_{m} m !} x^{m} .
$$

Using this formula, we get

$$
\begin{aligned}
& F_{3}\left(\alpha, \alpha^{\prime}, \beta, \beta^{\prime}, \gamma ; \frac{1}{x^{2}}, \frac{1}{1-x^{2}}\right) \\
&= \sum e^{-\pi i \lambda} \cos (\mu \pi) \frac{\Gamma(\gamma) \Gamma(\rho-\lambda) \Gamma(\sigma-\mu) \Gamma(\mu-\sigma+1) \Gamma(\gamma-\lambda-\mu-\sigma)}{\Gamma(\rho) \Gamma(\sigma) \Gamma(\gamma-\Lambda-\mu) \Gamma(1-\sigma) \Gamma(\gamma-\lambda-\sigma)} \\
& \times \quad x^{2 \lambda}\left(1-x^{2}\right)^{\gamma-\lambda-1}{ }_{3} F_{2}(\lambda+\mu-\gamma+1, \lambda+\sigma-\gamma+1,1-\rho ; \\
&\left.\lambda-\rho+1, \lambda+\mu+\sigma-\gamma+1 ; \frac{x^{2}}{x^{2}-1}\right) .
\end{aligned}
$$

In our case, the parameters $\left(\alpha, \alpha^{\prime}, \beta, \beta^{\prime}, \gamma\right)$ satisfy the following relation,

$$
\alpha+\alpha^{\prime}+\beta+\beta^{\prime}=\gamma
$$

so that we have

$$
1-\rho=\lambda+\mu+\sigma-\gamma+1
$$

Using this relation, we can find that the function reduces to the sum of hypergeometric functions;

$$
\begin{aligned}
& F_{3}\left(\alpha, \alpha^{\prime}, \beta, \beta^{\prime}, \gamma ; \frac{1}{x^{2}}, \frac{1}{1-x^{2}}\right) \\
= & e^{-\pi i \lambda} \frac{\cos \mu \pi \sin \sigma \pi}{\sin (\sigma-\mu) \pi} \frac{\Gamma(\gamma) \Gamma(\rho-\lambda)}{\Gamma(\rho+\sigma) \Gamma(\rho+\mu)} x^{2 \lambda}\left(1-x^{2}\right)^{\gamma-\lambda-1} \\
& \times F\left(1-\rho-\sigma, 1-\rho-\mu ; \lambda-\rho+1 ; \frac{x^{2}}{x^{2}-1}\right) .
\end{aligned}
$$

Making use of the relation;

$$
F(a, b ; c ; z)=(1-z)^{c-a-b} F(c-a, c-b ; c ; z)
$$


we finally obtain the formula

$$
\begin{aligned}
& F_{3}\left(\alpha, \alpha^{\prime}, \beta, \beta^{\prime}, \gamma ; \frac{1}{x^{2}}, \frac{1}{1-x^{2}}\right) \\
= & e^{-\pi i \alpha} \frac{\Gamma(\gamma) \Gamma(\beta-\alpha)}{\Gamma\left(\beta+\alpha^{\prime}\right) \Gamma\left(\beta+\beta^{\prime}\right)}\left(\frac{x^{2}}{1-x^{2}}\right)^{\lambda} F\left(\alpha+\alpha^{\prime}, \alpha+\beta^{\prime} ; \alpha-\beta+1 ; \frac{x^{2}}{x^{2}-1}\right) \\
& +(\alpha \leftrightarrow \beta), \quad\left(\text { for } \quad \alpha+\beta+\alpha^{\prime}+\beta^{\prime}=\gamma\right) .
\end{aligned}
$$

Next, we apply this formula to (4.9) and obtain

$$
\begin{aligned}
G_{l} & =e^{-\frac{\pi \omega}{2}}\left(1-x^{2}\right)^{-\frac{1}{2} l}\left[\frac{\Gamma\left(c+\frac{1}{2}\right) \Gamma\left(\frac{1}{2}\right)}{\Gamma\left(\frac{c}{2}+\frac{1}{2}+\frac{\nu}{2}\right) \Gamma\left(\frac{c}{2}+\frac{1}{2}-\frac{\nu}{2}\right)} F\left(\frac{c}{2}+\frac{\nu}{2}, \frac{c}{2}-\frac{\nu}{2} ; \frac{1}{2} ; \frac{x^{2}}{x^{2}-1}\right)\right. \\
& \left.+\frac{\Gamma\left(c+\frac{1}{2}\right) \Gamma\left(-\frac{1}{2}\right)}{\Gamma\left(\frac{c}{2}+\frac{\nu}{2}\right) \Gamma\left(\frac{c}{2}-\frac{\nu}{2}\right)} e^{-\frac{\pi}{2} i}\left(\frac{x^{2}}{1-x^{2}}\right)^{\frac{1}{2}} F\left(\frac{c}{2}+\frac{\nu}{2}+\frac{1}{2}, \frac{c}{2}-\frac{\nu}{2}+\frac{1}{2} ; \frac{3}{2} ; \frac{x^{2}}{x^{2}-1}\right)\right](4
\end{aligned}
$$

where we can observe the well-known factor $e^{-\frac{\pi \omega}{2}}$ [1] causing the Hawking radiation [8]. Furthermore, we can transform this function to a rather simple form by using the following formulae;

$$
\begin{aligned}
& \frac{2 \Gamma\left(\frac{1}{2}\right) \Gamma\left(a+b+\frac{1}{2}\right)}{\Gamma\left(a+\frac{1}{2}\right) \Gamma\left(b+\frac{1}{2}\right)} F\left(a, b ; \frac{1}{2} ; z\right) \\
= & F\left(2 a, 2 b ; a+b+\frac{1}{2} ; \frac{1}{2}\left(1+z^{\frac{1}{2}}\right)\right)+F\left(2 a, 2 b ; a+b+\frac{1}{2} ; \frac{1}{2}\left(1-z^{\frac{1}{2}}\right)\right), \\
& \frac{2 \Gamma\left(-\frac{1}{2}\right) \Gamma\left(a+b+\frac{1}{2}\right)}{\Gamma\left(a-\frac{1}{2}\right) \Gamma\left(b-\frac{1}{2}\right)} z^{\frac{1}{2}} F\left(a, b ; \frac{3}{2} ; z\right) \\
= & F\left(2 a-1,2 b-1 ; a+b-\frac{1}{2} ; \frac{1}{2}\left(1-z^{\frac{1}{2}}\right)\right) \\
& -F\left(2 a-1,2 b-1 ; a+b-\frac{1}{2} ; \frac{1}{2}\left(1+z^{\frac{1}{2}}\right)\right),
\end{aligned}
$$

as

$$
G_{l}=e^{-\frac{\pi \omega}{2}} \sin ^{-l} \eta_{2} F\left(l+\frac{3}{2}+\nu, l+\frac{3}{2}-\nu, l+2 ; \frac{i e^{-i \eta_{2}}}{2 \sin \eta_{2}}\right),
$$

and again using the formula (4.22) we get

$$
\begin{aligned}
G_{l} & =e^{-\frac{\pi \omega}{2}}\left(\sin \eta_{2}\right)(2 i)^{l+1} e^{-i(l+1) \eta_{2}} F\left(\nu+\frac{1}{2},-\nu+\frac{1}{2} ; l+2 ; \frac{i e^{-i \eta_{2}}}{2 \sin \eta_{2}}\right) \\
& =e^{-\frac{\pi \omega}{2}} \pi^{-\frac{1}{2}} 2^{l+\frac{3}{2}} i^{l+1} e^{\frac{i}{4} \pi-i \pi \nu} \frac{\Gamma(l+2)}{\Gamma\left(l+\nu+\frac{3}{2}\right)}\left(\sin \eta_{2}\right)^{\frac{3}{2}} Q_{l+\frac{1}{2}}^{\nu}\left(\cos \eta_{2}+i 0\right),
\end{aligned}
$$

Note that the term which depends on $\omega$ is just the factor $e^{-\frac{\pi \omega}{2}}$. 
Quite similarly, we can find that the next term of the Bogoliubov coefficient $G_{l+1}$ can be expressed as

$$
\begin{aligned}
G_{l+1}^{ \pm}= & \mp \frac{i \omega}{2(l+1)} x^{i \omega-1}\left(1-x^{2}\right)^{-\frac{1}{2}(l+i \omega)} \\
& \times F_{3}\left(-\frac{i \omega}{2}+\frac{1}{2}, a,-\frac{i \omega}{2}+1, b ; c+\frac{3}{2}, \frac{1}{x^{2}}, \frac{1}{1-x^{2}}\right) .
\end{aligned}
$$

By using the formula (4.23) again on this function, we obtain

$$
G_{l+1}^{ \pm}=\frac{i \omega}{l+1} e^{-\frac{\pi \omega}{2}} \pi^{-\frac{1}{2}} 2^{l+\frac{3}{2}} i^{l+1} e^{\frac{i}{4} \pi-i \pi \nu} \frac{\Gamma(l+3)}{\Gamma\left(l+\nu+\frac{5}{2}\right)}\left(\sin \eta_{2}\right)^{\frac{3}{2}} Q_{l+\frac{3}{2}}^{\nu}\left(\cos \eta_{2}+i 0\right) .
$$

We confirm the relation (4.5) comparing the coefficients of the mode functions on the RHS of (4.27) and (4.29).

\section{Discussions}

We have obtained Bogoliubov coefficient of the scalar wave functions from the global coordinates to the static coordinates. We considered two type of global coordinates, which we denoted type-I and type-II global coordinates.

Technically, we have used rather different formula for each case. For the type-I case, the Bogoliubov transformation corresponds to the Weber-Schafheitlin type integral, whereas we have used analytic continuation of the hypergeometric functions in two variables and proved a useful formula (4.23). The main difference of the correspondence of the Bogoliubov transformation between the type-I and the type-II coordinates stems from the use of the analytic continuations. In the type-I coordinates, we did not use any analytic continuation which is required for type-II coordinates. Unfortunately, we have not clarified the meaning of the analytic continuation. We do not know how to extract

the famous factor $e^{-\frac{\pi \omega}{2}}$ from the type-I case. To make sure the difference between these two coordinates, let us argue the relation of the mode functions in the type-I and type-II coordinates where the relation of the coordinates is given by

$$
-\eta_{1}=\frac{\sin \eta_{2}}{\cos \chi-\cos \eta_{2}}, \quad r_{1}=\frac{\sin \chi}{\cos \chi-\cos \eta_{2}}
$$


for $\cos \chi>\cos \eta_{2}$.

Since there seems to be any direct formula in this case, we start with the following integral formula 15 ;

$$
\begin{aligned}
& \int_{0}^{\infty} x^{\rho+\nu-\mu+1} J_{\mu}(x) J_{\nu}(\beta x) K_{\rho}(\gamma x) d x \\
= & 2^{\rho+\nu-\mu-1} \frac{\Gamma(\rho+\nu+1) \Gamma(\rho+1) \Gamma(\nu+1)}{\Gamma(\mu+1)}(\cosh \sigma-\cos \theta) \\
& \times P_{\rho+\nu-\mu}^{-\rho}(\cos \theta) P_{\rho+\nu-\mu}^{-\nu}(\cosh \sigma),
\end{aligned}
$$

where

$$
\beta=\frac{\sinh \sigma}{\cosh \sigma-\cos \theta}, \quad \gamma=\frac{\sin \theta}{\cosh \sigma-\cos \theta} .
$$

This integral is also Weber-Schafheitlin type. We could not find any proof of this formula in the literature. The proof may require some factorization formula of $F_{4}$ which is one of the hypergeometric function of two variables [13].

We will make an analytic continuation by $\sigma \rightarrow i \sigma$ keeping a relation $\cos \theta<\cos \sigma$, intrinsic to discontinuous integral. Then we have

$$
\begin{aligned}
& \int_{0}^{\infty} x^{\rho+\nu-\mu+1} J_{\mu}(x) I_{\nu}(\beta x) K_{\rho}(\gamma x) d x \\
= & 2^{\rho+\nu-\mu-1} \frac{\Gamma(\rho+\nu+1) \Gamma(\rho+1) \Gamma(\nu+1)}{\Gamma(\mu+1)}(\cos \sigma-\cos \theta) \\
& \times P_{\rho+\nu-\mu}^{-\rho}(\cos \theta) P_{\rho+\nu-\mu}^{-\nu}(\cos \sigma),
\end{aligned}
$$

where

$$
\beta=\frac{\sin \sigma}{\cos \sigma-\cos \theta}, \quad \gamma=\frac{\sin \theta}{\cos \sigma-\cos \theta} .
$$

In the integral, we will use another integral formula [16]

$$
\begin{aligned}
I_{\nu}(\beta x) K_{\rho}(\gamma x)= & \int_{0}^{\infty} d t \frac{x^{-\lambda} t^{\lambda+1}}{t^{2}+x^{2}}\left\{\cos \left[\frac{1}{2}(\lambda-\rho+\nu) \pi\right] J_{\rho}(\gamma t)\right. \\
& \left.+\sin \left[\frac{1}{2}(\lambda-\rho+\nu) \pi\right] Y_{\rho}(\gamma t)\right\} J_{\nu}(\beta t),
\end{aligned}
$$

and setting $\lambda=\rho+\nu-2 \mu$, performing integration over $x$ by using a formula [17], we have the following integral formula

$$
\int_{0}^{\infty} d k k^{N+\frac{1}{2}} K_{-\nu-N+l}(k)\left\{\cos \left[\left(N+\nu+\frac{1}{2}\right) \pi\right]\right\} J_{-\nu}\left(-k \eta_{1}\right)
$$




$$
\begin{gathered}
\left.+\sin \left[\left(N+\nu+\frac{1}{2}\right) \pi\right] Y_{-\nu}\left(-k \eta_{1}\right)\right\}\left(-k \eta_{1}\right)^{\frac{3}{2}} J_{l+\frac{1}{2}}\left(k r_{1}\right)\left(k r_{1}\right)^{-\frac{1}{2}} \\
=2^{N-l-1}(-1)^{-\frac{1}{2} l-\frac{1}{4}} \frac{\Gamma\left(l-\nu+\frac{3}{2}\right) \Gamma(-\nu+1) \Gamma(2 l+2) \Gamma(N-l+1)}{\Gamma(-\nu-N+l+1) \Gamma(N+l+2)} \\
\times\left(\sin \eta_{2}\right)^{\frac{3}{2}} P_{N+\frac{1}{2}}^{\nu}\left(\cos \eta_{2}\right)(\sin \chi)^{l} C_{N-l}^{l+1}(\cos \chi) .
\end{gathered}
$$

Unfortunately, from this kind of Bogoliubov transformation, we can not fix the Bogoliubov transformation uniquely because there are many vanishing identities in the integral formula. As a matter of fact, we can change the ratio of $J_{\rho}$ and $Y_{\rho}$ by the choice of the parameter $\lambda$ in (5.6). Therefore we are required to consider the inverse transformation of (5.7) in order to fix the coefficient, which we fail to perform. Anyway, the formula (5.7) shows an existence of the Bogoliubov transformation between the mode functions of global coordinates, which is expected because we know the Green functions for both global coordinates are identical.

In this paper, we have obtained the Bogoliubov coefficients from global coordinates to the static coordinates. Although we have shown that the Bogoliubov transformations are related to some mathematical formula including those of hypergeometric functions in two variables, the true physical interpretation of the analytic continuation is not clear, which deserves further investigation. 


\section{References}

[1] S.W.Hawking, Commun.Math.Phys. 43 (1975) 199.

[2] O.Nachtmann, Commn.Math.Phys. 6 (1967) 1.

[3] N.A. Chernikow and E.A. Tagirov, Ann. Inst. Henri Poincaré, 9A (1968) 109.

[4] D. Rohiya and N. Panchapakesan, J. Phys. A: Gen.Phys., 11 (1978) 1963.

[5] E.A. Tagirov, Ann. Phys. 76 (1973) 561.

[6] G.W. Gibbons and S.W. Hawining, Phys. Rev. D15 (1977) 2738.

[7] W.G.Unruh, Phys.Rev.D14(1976) 870.

[8] A.S. Lapedes, J. Math. Phys. 19 (1987) 2289.

[9] “Higher Transcendental Functions," eds. A.Erdélyi et al., McGraw-Hill, New York (1953) Vol.II, p 51 (29).

[10] "Higher Transcendental Functions," eds. A.Erdélyi et al., McGraw-Hill, New York (1953) Vol.I, p 101.

[11] “ Higher Transcendental Functions," eds. A.Erdélyi et al., McGraw-Hill, New York (1953) Vol.I, p.231,(7).

[12] P.Appell and M.J.Kampé de Fériet, "Fonctions hypergéométriques et hypersphériques," Polymones d'Hermite, Gauthier-Villars (1926);

A. Erdélyi, Proc. Roy. Soc. Edinburgh Sect. A62 (1948) pp.378-385;

"Higher Transcendental Functions," eds. A.Erdélyi et al., McGraw-Hill, New York (1953) Vol.I, p.241,(11).

[13] "Higher Transcendental Functions," eds. A.Erdélyi et al., McGraw-Hill, New York (1953) Vol.I. p 224. 
[14] "Higher Transcendental Functions," eds. A.Erdélyi et al., McGraw-Hill, New York (1953) Vol.I. p.240,(6).

[15] "Table of Integral Transforms," eds. A.Erdélyi et al., McGraw-Hill, New York (1954) Vol.II, p65,(14).

[16] "Table of Integral Transforms," eds. A.Erdélyi et al., McGraw-Hill, New York (1954) Vol.II, p55,(47).

[17] "Table of Integral Transforms," eds. A.Erdélyi et al., McGraw-Hill, New York (1954)Vol.II, p23,(12). 